\title{
Sound field reconstruction using acousto-optic tomography
}

\author{
Torras Rosell, Antoni; Barrera Figueroa, Salvador; Jacobsen, Finn
}

Published in:

Acoustical Society of America. Journal

Link to article, DOI:

$10.1121 / 1.3695394$

Publication date:

2012

Document Version

Publisher's PDF, also known as Version of record

Link back to DTU Orbit

Citation (APA):

Torras Rosell, A., Barrera Figueroa, S., \& Jacobsen, F. (2012). Sound field reconstruction using acousto-optic tomography. Acoustical Society of America. Journal, 131(5), 3786-3793. https://doi.org/10.1121/1.3695394

\section{General rights}

Copyright and moral rights for the publications made accessible in the public portal are retained by the authors and/or other copyright owners and it is a condition of accessing publications that users recognise and abide by the legal requirements associated with these rights.

- Users may download and print one copy of any publication from the public portal for the purpose of private study or research.

- You may not further distribute the material or use it for any profit-making activity or commercial gain

- You may freely distribute the URL identifying the publication in the public portal

If you believe that this document breaches copyright please contact us providing details, and we will remove access to the work immediately and investigate your claim. 


\title{
Sound field reconstruction using acousto-optic tomography ${ }^{\text {a) }}$
}

\author{
Antoni Torras-Rosell ${ }^{\text {b) }}$ and Salvador Barrera-Figueroa \\ Danish Fundamental Metrology A/S, Matematiktorvet 307, 2800 Kongens Lyngby, Denmark \\ Finn Jacobsen \\ Acoustic Technology, Department of Electrical Engineering, Technical University of Denmark, Ørsteds Plads \\ 352, 2800 Kongens Lyngby, Denmark
}

(Received 28 September 2011; revised 15 February 2012; accepted 19 February 2012)

\begin{abstract}
When sound propagates through a medium, it results in pressure fluctuations that change the instantaneous density of the medium. Under such circumstances, the refractive index that characterizes the propagation of light is not constant, but influenced by the acoustic field. This kind of interaction is known as the acousto-optic effect. The formulation of this physical phenomenon into a mathematical problem can be described in terms of the Radon transform, which makes it possible to reconstruct an arbitrary sound field using tomography. The present work derives the fundamental equations governing the acousto-optic effect in air, and demonstrates that it can be measured with a laser Doppler vibrometer in the audible frequency range. The tomographic reconstruction is tested by means of computer simulations and measurements. The main features observed in the simulations are also recognized in the experimental results. The effectiveness of the tomographic reconstruction is further confirmed with representations of the very same sound field measured with a traditional microphone array. (C) 2012 Acoustical Society of America. [http://dx.doi.org/10.1121/1.3695394]
\end{abstract}

PACS number(s): 43.35.Sx [JDM]

Pages: $3786-3793$

\section{INTRODUCTION}

The acousto-optic effect has been extensively used to characterize ultrasonic waves in underwater acoustics. ${ }^{1-7}$ In such measurements, the acoustic properties of sound are determined by measuring the small changes of the refractive index that are induced by the pressure fluctuations of the acoustic field. These variations of the refractive index will cause diffraction and changes of the speed of light that influence the propagation of light in amplitude and phase. In practice, in the low ultrasonic frequency range, diffraction effects can be neglected when the acoustic field has small amplitudes. ${ }^{2,6,7}$

Ultrasonic measurements in air are not commonly based on the acousto-optic effect (although some examples can be found in Refs. 8 and 9). This is perhaps because ultrasound is highly attenuated in air and because there normally is a significant impedance mismatch between the air and the ultrasonic transducer. ${ }^{8}$ These constraints are less severe when using conventional loudspeakers and microphones in the audible frequency range. However, only a few investigations have been reported for audible sound, ${ }^{10-12}$ and they have been concerned with visualization purposes rather than quantification.

The aim of this work is to demonstrate that light can be used as a means to characterize airborne sound in the audible frequency range. First, the physical principles governing the acousto-optic effect are presented. This will show that sound

\footnotetext{
a) Portions of this work were presented in "Sound field reconstruction based on the acousto-optic effect," Proceedings of Inter-Noise 2011, Osaka, Japan, September 2011.

b) Author to whom correspondence should be addressed. Electronic mail: atr@dfm.dtu.dk
}

pressure fluctuations are captured in the phase of a light beam that travels through the medium. Next, we describe the measurement procedure used to retrieve the phase of the light as an apparent velocity measured with a laser Doppler vibrometer (LDV). This apparent velocity is interpreted as a projection of the sound field. Several projections in different directions can be used to reconstruct the acoustic field using tomography. The quality of the tomographic reconstruction is finally assessed by means of simulations and experimental results.

\section{ACOUSTO-OPTIC TOMOGRAPHY}

\section{A. Acousto-optic effect}

The phenomenon of sound inherently involves pressure fluctuations that change the density of the medium. Assuming adiabatic conditions, the total pressure $p_{t}$ and the density $\rho$ of the medium are related by means of the following expression: ${ }^{13}$

$$
\frac{p_{t}}{p_{0}}=\left(\frac{\rho}{\rho_{0}}\right)^{\gamma}
$$

where $p_{0}$ and $\rho_{0}$ are the pressure and the density under static conditions, and $\gamma$ is the ratio of specific heats. Note that, when sound propagates, $p_{t}$ corresponds to the superposition of the static and the acoustic pressures, that is, $p_{t}=p_{0}+p$. The influence of the density variations on the propagation of light can be determined by combining the mechanical and optical properties of the medium. In 1863, Gladstone and Dale established an empirical relation between the refractive index $n$ and the density $\rho$ of various liquids ${ }^{14}$

$$
n-1=G \rho,
$$


where the Gladstone-Dale constant $G$ is an intrinsic feature of the liquid. This relation also holds for air. ${ }^{15}$ The most important property of the latter expression is not the exact value of $G$, but the fact that density and refractive index exhibit a linear relationship. The relation between the refractive index and the acoustic field can be established by combining Eqs. (1) and (2)

$$
n=\left(n_{0}-1\right)\left(\frac{p_{t}}{p_{0}}\right)^{1 / \gamma}+1=\left(n_{0}-1\right)\left(1+\frac{p}{p_{0}}\right)^{1 / \gamma}+1,
$$

where $n_{0}$ is the index of refraction under standard atmospheric conditions. As shown in Appendix A, this expression can be approximated by a first order Taylor expansion when the acoustic pressure is much smaller than the static pressure, $p \ll p_{0}$,

$$
n \cong n_{0}+\frac{n_{0}-1}{\gamma p_{0}} p .
$$

Thus, under weak acousto-optic interaction, the variations of the refractive index are proportional to the sound pressure. For ease of reference, a sound pressure of $1 \mathrm{~Pa}$ yields an increase of the refractive index of air of about $2 \times 10^{-9}$ of its value under static conditions.

\section{B. Measurement principle}

The understanding of the physical phenomenon governing the acousto-optic effect opens up the possibility of characterizing sound by measuring light that travels through an acoustic field. In this context, light can be regarded as an electromagnetic wave $\mathbf{E}$ that satisfies the electromagnetic wave equation

$$
\nabla^{2} \mathbf{E}-\left(\frac{n}{c_{0}}\right)^{2} \frac{\partial^{2} \mathbf{E}}{\partial t^{2}}=0
$$

where $c_{0}$ corresponds to the speed of light in vacuum. Although this fundamental equation is normally derived for waves propagating through a homogeneous quiescent medium (which is not completely true in the presence of sound), correct solutions can still be obtained when the following condition is fulfilled: ${ }^{16}$

$$
\left|\frac{1}{n} \frac{\partial n}{\partial t} T\right| \ll 1
$$

where $T$ corresponds to the oscillation period of the electric field. This is indeed the case for weak acousto-optic interaction (see Appendix B for further details). Under such conditions, the acousto-optic effect modifies the phase of light rather than its amplitude. One can think of it as an electromagnetic wave that travels faster or slower depending on the pressure fluctuations caused by the acoustic field. The light travels slower when the medium is denser (pressure increase) and faster otherwise. These ideas bring up the possibility of using the following expression as a solution to the electromagnetic wave equation in the presence of sound

$$
\mathbf{E}=\mathbf{E}_{0} e^{j\left(\omega_{o} t+\phi(x, y, z, t)\right)},
$$

where $\omega_{o}$ is the angular frequency of the light, $\mathbf{E}_{0}$ is a complex number that accounts for the amplitude and the polarization of the light, and $\phi$ is the phase term that depends on the acousto-optic effect. As demonstrated in Appendix B, the general solution of $\phi$ resulting from inserting Eq. (7) into the wave equation is

$$
\phi=k_{0} \int_{\mathbf{L}} n \mathrm{~d} l=k_{0} n_{0} L_{0}+k_{0} \frac{n_{0}-1}{\gamma p_{0}} \int_{\mathbf{L}} p(x, y, z, t) \mathrm{d} l,
$$

where $k_{0}$ is the wave number of light in vacuum, $\mathbf{L}$ represents the path followed by the light, and $L_{0}$ is the corresponding distance. It is worth noting that in the absence of sound, $\phi=k_{0} n_{0} L_{0}$, that is, the classical phase shift of a plane wave propagating in a homogeneous quiescent medium. Equation (8) establishes that the phase of a beam of light that travels through an acoustic field is proportional to the line integral of the sound pressure. This can be exploited as a measurement principle for acoustic measurements, but it requires measuring the phase of the light accurately. An LDV can effectively provide this information. In conventional use, the vibrational velocity of a surface measured by the LDV is proportional to the time derivative of the phase of the light

$$
v(t)=\frac{1}{k_{0} n_{0}} \frac{\mathrm{d} \phi}{\mathrm{d} t} .
$$

In the absence of sound $\left(\phi=k_{0} n_{0} L_{0}\right)$, the velocity retrieved by an LDV is equal to the rate of change of the distance from the head of the LDV to the reflecting point where the laser is pointed, that is, $v(t)=\mathrm{d} L_{0} / \mathrm{d} t$. However, an LDV can also measure the acousto-optic effect when the vibrations of the surface that reflects the light back to the LDV are negligible. ${ }^{17}$ In such a case, $\mathrm{d} L_{0} / \mathrm{d} t \approx 0$ and the acousto-optic effect [the second term on the right hand side of Eq. (8)] dominates in the time derivative of $\phi$

$$
v(t)=\frac{n_{0}-1}{\gamma p_{0} n_{0}} \frac{\mathrm{d}}{\mathrm{d} t}\left(\int_{\mathbf{L}} p(x, y, z, t) \mathrm{d} l\right) .
$$

\section{Sound pressure reconstruction}

The line integral of the acoustic pressure can be seen as a projection of the sound field in the direction of propagation of the light. These projections can be described mathematically by means of the Radon transform ${ }^{18}$

$$
R_{p}\left(x^{\prime}, \theta, t\right)=\int_{-\infty}^{+\infty} p(x, y, t) \mathrm{d} y^{\prime},
$$

where the coordinates $x^{\prime}$ and $y^{\prime}$ are related to the coordinates $x$ and $y$ by means of the following rotation matrix:

$$
\left[\begin{array}{l}
x^{\prime} \\
y^{\prime}
\end{array}\right]=\left[\begin{array}{cc}
\cos \theta & \sin \theta \\
-\sin \theta & \cos \theta
\end{array}\right]\left[\begin{array}{l}
x \\
y
\end{array}\right]
$$


This coordinate system transformation is illustrated in Fig. 1. It is easy to see from Eq. (10) that the Radon transform of the sound field under investigation can be obtained from the apparent velocity of the LDV as follows

$$
R_{p}\left(x^{\prime}, \theta, t\right)=\frac{\gamma p_{0} n_{0}}{n_{0}-1} \int v\left(x^{\prime}, \theta, t\right) \mathrm{d} t
$$

Unless assumptions about the symmetry of the sound field are made, the information obtained from a single line scan is, in general, not sufficient to reconstruct the acoustic field. ${ }^{17}$ The reconstruction of an arbitrary sound field requires the use of tomography. The sound field is first scanned over a plane, e.g., along a set of parallel lines as indicated in Fig. 1, and this procedure must then be repeated (synchronously) for a series of angles of $\theta$ from $0^{\circ}$ to $180^{\circ}$. It is not necessary to rotate up to $360^{\circ}$ when using a parallel beam scan configuration because the projections obtained for angles $\theta \geq 180^{\circ}$ are replicas of the scans measured at $\theta-180^{\circ}$. With this procedure, the acoustic field is projected into different directions, and thus, the resulting data set constitutes a well-defined inverse problem that can be solved by means of the inverse Radon transform. The latter can in practice be implemented efficiently by means of the filtered backprojection algorithm, ${ }^{18}$ which in a parallel beam scan configuration can be defined as

$$
\tilde{p}(x, y, t)=\int_{0}^{\pi} Q\left(x^{\prime}, \theta, t\right) \mathrm{d} \theta,
$$

where $\tilde{p}(x, y, t)$ denotes the reconstructed sound pressure, and $Q\left(x^{\prime}, \theta, t\right)$ is the so-called "filtered projection"

$$
Q\left(x^{\prime}, \theta, t\right)=R_{p}\left(x^{\prime}, \theta, t\right) * h\left(x^{\prime}\right),
$$

that is, the convolution of the measured $R_{p}\left(x^{\prime}, \theta, t\right)$ with a filter $h\left(x^{\prime}\right)$ that accounts for both the filtering required to implement the algorithm with a two-dimensional (2D) spatial

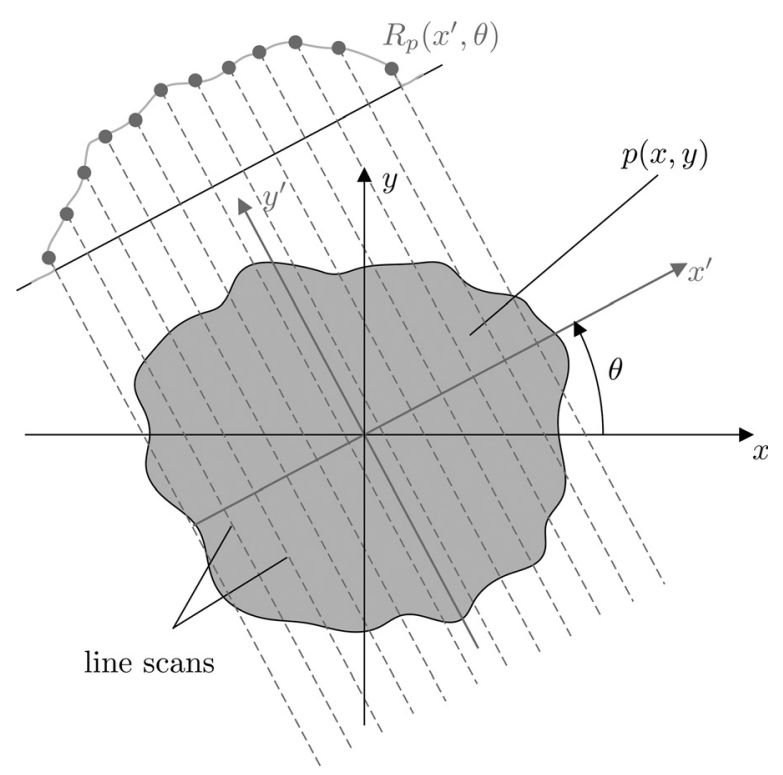

FIG. 1. Sketch of the parallel beam scan setup for obtaining the Radon transform of an acoustic field.
Fourier transform (Ram-Lak filter) and the windowing imposed in the frequency domain in order to reduce the influence of noise outside of the frequency range of interest (e.g., a Hann or a Cosine window). Note that the phase of the light performs a continuous integration of the sound field, not a discrete summation. However, the data set used in the reconstruction algorithm consists of a finite number of samples. This means that one must either estimate the continuous integrals of the inverse Radon transform with numerical approximations or use the inverse discrete Radon transform. The latter option does not account for the spatial resolution used in the measurements. This biases the amplitude of the reconstructed pressure, but it can easily be corrected by dividing the reconstructed pressure by the spatial resolution. In either case, the quality of the reconstruction depends on the spatial and angular resolutions used to sample the Radon transform of the acoustic field.

\section{SIMULATION AND EXPERIMENTAL RESULTS}

\section{A. Simulations}

The interpretation of the acousto-optic effect as the Radon transform of an acoustic field is analyzed by means of a computer simulation. For clarity's sake, the case of study is a monopole that radiates sound at a frequency of $2 \mathrm{kHz}$. Figure 2(a) shows the instantaneous spherical sound field emitted by the monopole in a plane at a distance of $12 \mathrm{~cm}$ from the point source. Figure 2(b) shows the corresponding Radon transform as a function of $x^{\prime}$ and $\theta$. As can be seen, the Radon transform does not change with the angle of rotation $\theta$. This is a consequence of the spherical symmetry of the sound field under study, i.e., the integral of the acoustic field leads to the same result independently of $\theta$. As mentioned previously, it is only possible in practice to measure a discrete number of points of the Radon transform, and thus, the quality of the reconstruction relies on the separation between the line scans (spatial resolution) and the angular resolution used to rotate the measurement setup in order to scan the sound field in different directions. Figure 3(a) shows the reconstructed sound field when the spatial and angular resolutions are $1 \mathrm{~cm}$ and $1^{\circ}$, and Fig. 3(b) corresponds to the spatial and angular resolutions of $4 \mathrm{~cm}$ and $10^{\circ}$. As expected, the reconstruction deteriorates when the spatial/angular resolution is coarser, that is, when the number of samples of the Radon transform that are used to compute the inverse transform is reduced.

\section{B. Measurements}

For ease of comparison with the simulations presented in Sec. III A, measurements were carried out in an anechoic room of about $1000 \mathrm{~m}^{3}$ and the sound field was generated by a loudspeaker driven with a pure tone of $2 \mathrm{kHz}$. The signal emitted by the loudspeaker was synchronized with the data acquisition system, allowing for the reconstruction of the instantaneous sound field. The measuring plane was located $12 \mathrm{~cm}$ above the loudspeaker. A picture of the measurement setup with an LDV Type OFV-505 manufactured by Polytec (Waldbronn, Germany) can be seen in Fig. 4(a). Instead of rotating the LDV 


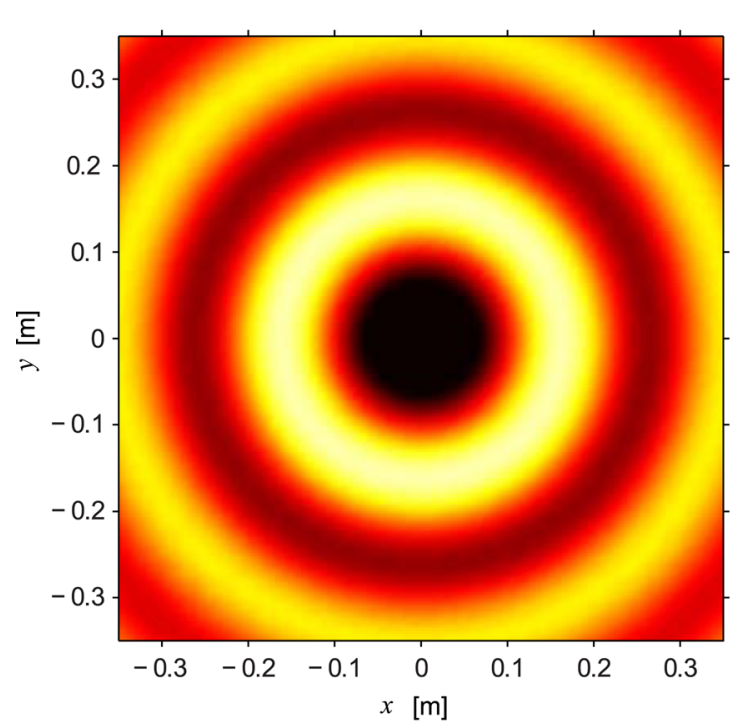

(a) Instantaneous sound field.

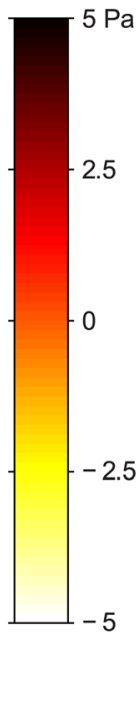

2.5

FIG. 2. (Color online) Monopole located $12 \mathrm{~cm}$ away from the simulated plane. together with the reflecting point, the loudspeaker was placed on a turntable allowing the scanning of the sound field in several directions. In this way, the structure where the LDV and the reflecting point were mounted was more stable. The turntable was moved manually in the perpendicular direction of the light beam allowing scanning in parallel lines. Besides, an accelerometer manufactured by Brüel \& Kjær (B\&K, Nærum, Denmark) Type 4344 was installed on top of the reflecting point in order to monitor that its acceleration was negligible, and thus, ensuring that the velocity output of the LDV was essentially caused by the acousto-optic effect. The outputs of the LDV and the accelerometer were captured with an external sound card Type HDSPe ExpressCard manufactured by RME (Haimhausen, Germany) that was controlled with a laptop by

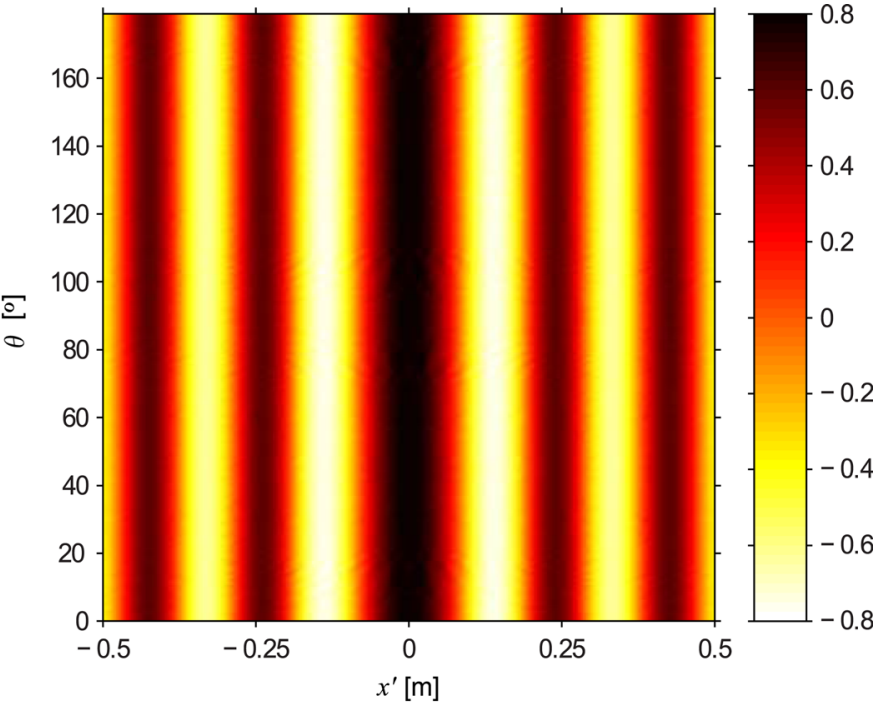

(b) Instantaneous Radon transform. means of a homemade Matlab program. The measured data was afterwards also analyzed with Matlab.

The Radon transform of the acoustic field generated by the loudspeaker was computed from the velocity measured with the LDV according to Eq. (13). The result obtained when using a spatial resolution of $2 \mathrm{~cm}$ and an angular resolution of $10^{\circ}$ is depicted in Fig. 4(b). Similar to the simulation presented in Sec. III A, the pattern of vertical lines exhibited by the Radon transform indicates that the measured Radon transform is fairly independent of $\theta$, which means that the reconstructed sound field should be rather rotationally symmetric. The pressure reconstructed after applying the inverse transform is presented in Fig. 5(a). As expected, the resulting sound field is spherically symmetric

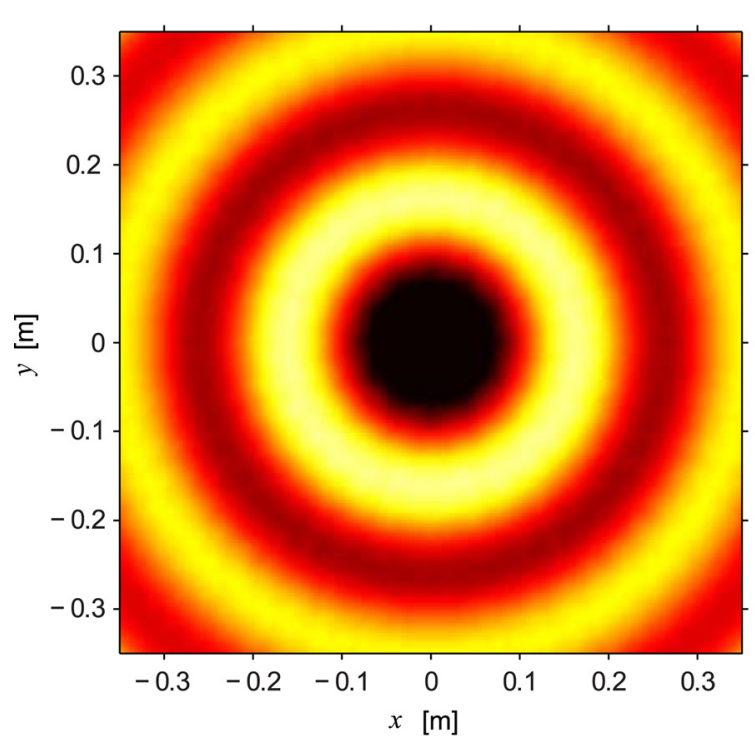

(a) Spatial resolution $1 \mathrm{~cm}$, angular resolution $1^{\circ}$.

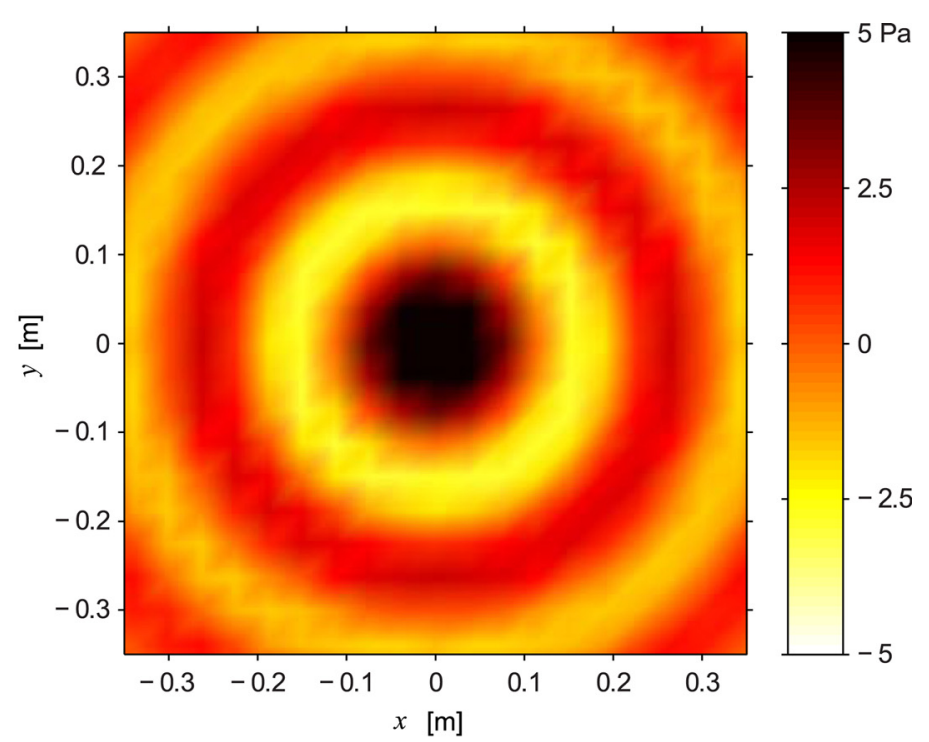

(b) Spatial resolution $4 \mathrm{~cm}$, angular resolution $10^{\circ}$.

FIG. 3. (Color online) Simulation of the tomographic reconstruction of the instantaneous sound field generated by the monopole. The filtered backprojection algorithm included a Lam-Rak filter and a Cosine window. 


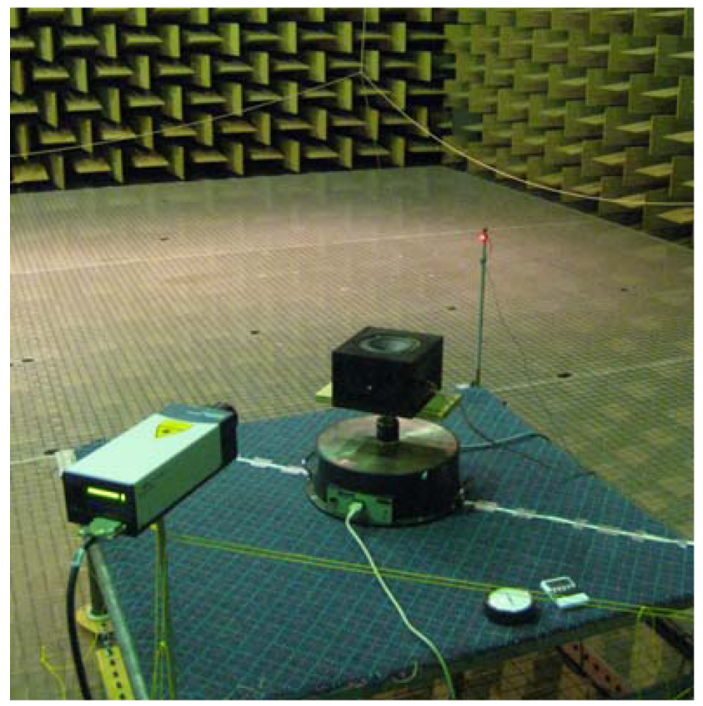

(a) LDV setup.

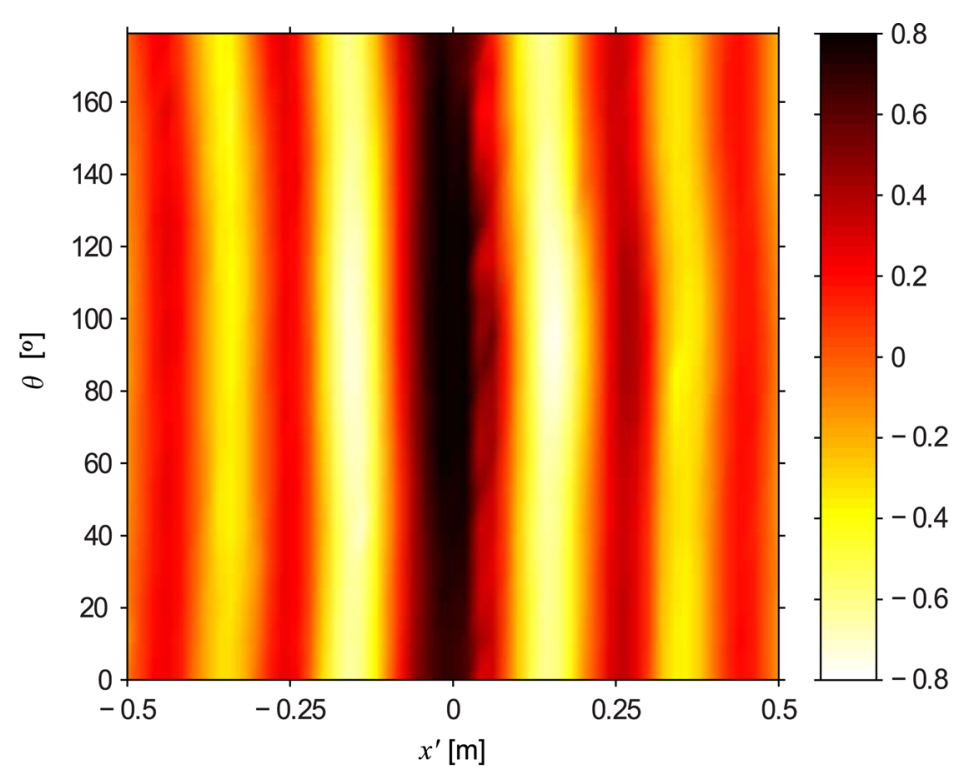

(b) Instantaneous Radon transform of the measured acoustic field.

FIG. 4. (Color online) Measurement of the Radon transform of the sound field with an LDV.

and exhibits the periodicities of a $2 \mathrm{kHz}$ pure tone (cf. the simulation results presented in Fig. 3). Figure 5(b) shows the reconstructed field when the spatial and angular resolutions are $4 \mathrm{~cm}$ and $10^{\circ}$, that is, with half of the line scans of the previous reconstruction. As observed in the simulations, the result becomes more blurred when decreasing the number of samples of the Radon transform used to reconstruct the sound field.

Finally, complementary measurements were carried out with a microphone array in order to compare the tomographic technique with a more traditional and well-established method. Figure 6(a) shows the planar rectangular microphone array used in this measurement. The array consisted of sixty $1 / 4$ in. microphones (B\&K Type 4957) with a spacing of 7.5 $\mathrm{cm}$ between the microphones. The measured sound field can be seen in Fig. 6(b). Despite the use of 60 microphones, the spatial resolution of the sampled sound field $(7.5 \mathrm{~cm})$ and the covered area $\left(67.5 \times 37.5 \mathrm{~cm}^{2}\right)$ are not directly comparable to the ones obtained with the tomographic technique. However, the overall amplitudes and pressure distribution are in fairly good agreement with the reconstructed fields shown in Fig. 5.

\section{DISCUSSION}

The figures presented in Sec. III represent a single instant of time of the simulated/measured Radon transforms and the corresponding instantaneous pressure reconstructions. They do not represent an average quantity. In fact, the complete output of the tomographic algorithm is a time series of the sound pressure measured over the area scanned

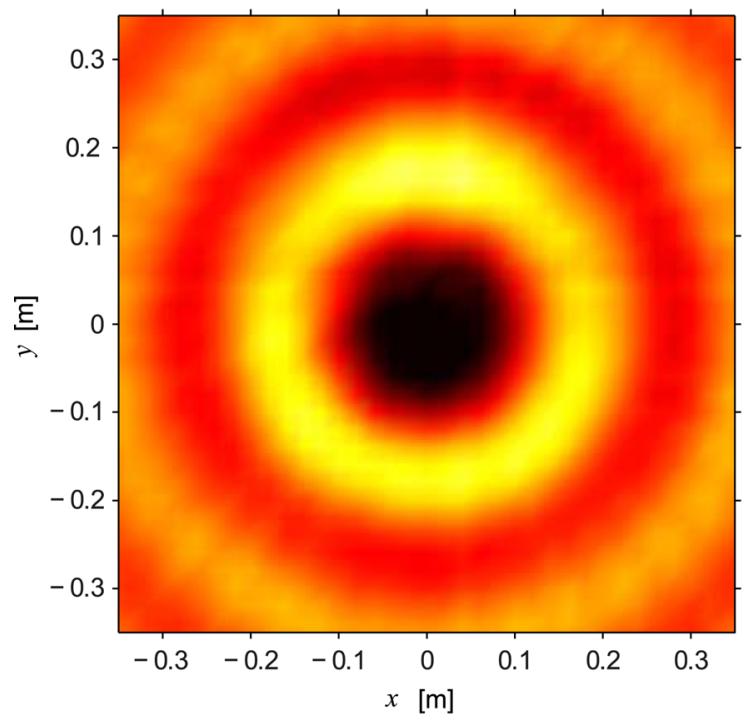

(a) Spatial resolution $2 \mathrm{~cm}$, angular resolution $10^{\circ}$.

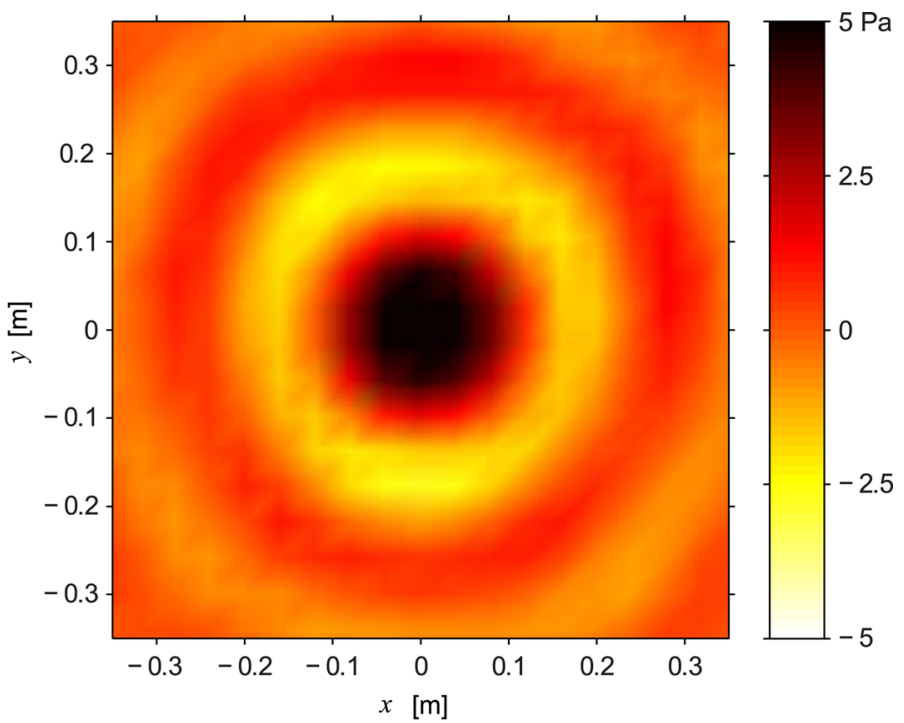

(b) Spatial resolution $4 \mathrm{~cm}$, angular resolution $10^{\circ}$.

FIG. 5. (Color online) Tomographic reconstruction of the instantaneous sound field radiated by the loudspeaker. The filtered backprojection algorithm included a Lam-Rak filter and a Cosine window. 


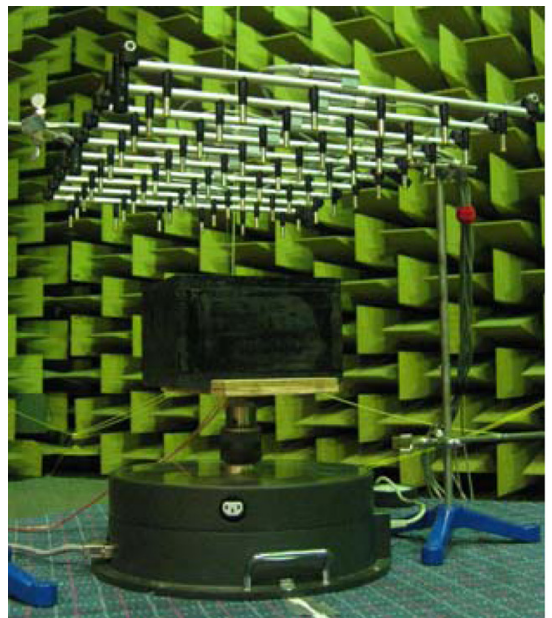

(a) Microphone array setup.

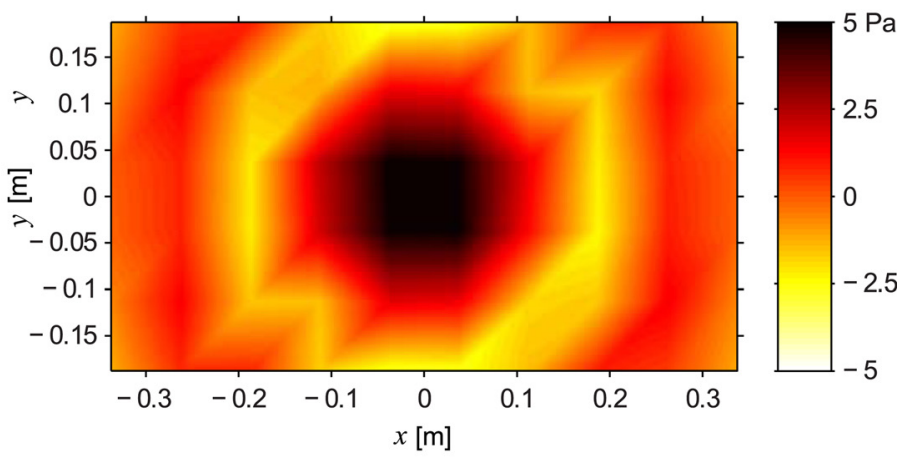

(b) Measured instantaneous pressure.

FIG. 6. (Color online) Sound field measurement with a planar rectangular microphone array.

with the LDV. This can serve the purposes of many sound visualization applications that are implemented nowadays with microphone arrays. Besides, sound visualization based on acousto-optic tomography has the great advantage of being a non-invasive technique. The presence of a large number of transducers often limits the frequency range that can be covered with conventional measurement systems. Increasing the number of transducers in order to enhance the spatial resolution of the array tends to aggravate the influence of the array on the quantities measured at high frequencies. A transducer-based correction in form of a frequency response can be applied at a post-processing stage to counterbalance this effect. There are also some transducers that are specifically designed to compensate for it. Nevertheless, the applied correction will only be valid for a certain direction of incident sound, typically axial incidence. Alternatively, one can, for instance, improve the grid resolution of a microphone array (without increasing the number of transducers) by performing synchronous measurements where the array is simply shifted, say, half of the spacing between transducers. In any case, it is necessary to immerse the array into the acoustic field. This is not the case when using the light as a "sensing" element.

The spatial resolution is also an important issue when using acousto-optic tomography. In spite of the continuous Radon transform measured with the LDV, only a finite number of line scans are captured for each direction of projection $\theta$. Therefore, as in any other discretization problem, aliasing effects may arise if the Nyquist theorem is not fulfilled - the spatial resolution should always be smaller than half the smallest wavelength of interest. Furthermore, it is also recommendable to have roughly as many directions of projection as parallel scans per projection. ${ }^{18}$

The available technology for measuring the acoustooptic effect has not been constructed for this purpose. The LDV is a device specially designed to measure mechanical vibrations, and besides, a feasible measurement setup currently requires one to measure the sound field in a synchronous fashion. Nevertheless, the results achieved with such an off-the-shelf technology are quite satisfactory. Further inves- tigations in this field could eventually lead to a new type of optical device capable of measuring sound from the interaction with light in real time. Thus, acousto-optic tomography may have a great potential in acoustical holography and beamforming.

The present work examines the interaction between sound and light as a means to visualize acoustic fields. However, the refractive index, and thus, the propagation of light can also be perturbed by other phenomena, e.g., the presence of flow or temperature gradients. This could limit the number of applications for acousto-optic tomography. Nevertheless, further research needs to be done in this direction and perhaps new acousto-optic techniques will be developed to compensate for these effects.

\section{CONCLUSIONS}

The interaction between sound and light has been investigated as a means to characterize airborne sound fields in the audible frequency range. Both simulations and measurements show that the acousto-optic effect can be used to reconstruct an acoustic field using tomography. Despite the technological limitations of the measurement setup, the experimental results demonstrate that instantaneous pressure fluctuations of an acoustic field can be captured with an LDV. The apparent velocity caused by the acousto-optic effect can be interpreted as projections of the acoustic field, which can be described mathematically by means of the Radon transform. Scanning the sound field in parallel lines and projecting it in different directions constitute an inverse problem that can be solved by means of the inverse Radon transform. The results show that the quality of the reconstructed sound field depends on the spatial and angular resolutions used during the measurement. Nevertheless, satisfactory results can be obtained without very fine resolution, especially when considering the number of microphones that would be required to map the sound field with a similar spatial resolution. Furthermore, the use of light as a sensing element (instead of a bulk instrument) makes the acousto-optic effect a non-invasive technique. This together with the fact that the tomographic reconstruction does not 
assume anything about the properties of the acoustic field turns the acousto-optic effect into a very attractive measurement principle for the visualization of sound fields.

\section{APPENDIX A: TAYLOR SERIES OF THE REFRACTIVE INDEX}

The refractive index changes slightly with the pressure fluctuations caused by an acoustic field. The small magnitude of these variations makes it possible to approximate Eq. (3) with a Taylor expansion. If Eq. (3) is rewritten as

$$
n(x)=\left(n_{0}-1\right)(1+x)^{1 / \gamma}+1,
$$

where $x$ corresponds to $p / p_{0}$, then the corresponding Taylor series can be calculated from the following expression:

$$
n(x)=\sum_{i=0}^{\infty} \frac{n^{(i)}(0)}{i !} x^{i}
$$

where $n^{(i)}(0)$ is the $i$ th derivative of the refractive index evaluated at zero. Note that the Taylor series is centered at zero because $x$ fluctuates around this value. Furthermore, the infinite summation stated in Eq. (A2) can in practice be truncated when $|x| \ll 1$. This is indeed the case since the amplitude of the acoustic pressure is much smaller than the value of the static pressure $\left(|p| \ll p_{0}\right)$. Therefore,

$$
\begin{aligned}
n(x) \simeq n(0)+\frac{n^{\prime}(0)}{1 !} x^{1} & =n(0)+n^{\prime}(0) x \\
& =n_{0}+\frac{n_{0}-1}{\gamma} x \quad \text { for } \quad|x| \ll 1,
\end{aligned}
$$

where $n(0)$ and $n^{\prime}(0)$ have been determined from Eq. (A1). Finally, substituting $x$ by the ratio of the acoustic pressure to the atmospheric pressure yields the approximation of the index of refraction given by Eq. (4).

\section{APPENDIX B: SOLUTION OF THE ELECTROMAG- NETIC WAVE EQUATION}

Equation (6) states the condition required in order to guarantee the validity of the solutions obtained from the electromagnetic wave equation in the presence of disturbing sound. Under weak acousto-optic interaction, the magnitude of the variations of the refractive index is very small, and in particular, the overall magnitude of the refractive index can be regarded as very close to unity when the light propagates in air. Moreover, the time derivative of the refractive index is proportional to the angular frequency of oscillation of the acoustic field. Thus, Eq. (6) can be reformulated as

$$
\left.\begin{array}{c}
n \approx 1 \\
\frac{\partial n}{\partial t} \propto \omega_{a} \\
T=\frac{2 \pi}{\omega_{o}}
\end{array}\right\} \Rightarrow\left|\frac{1}{n} \frac{\partial n}{\partial t} T\right| \approx\left|2 \pi \frac{\omega_{a}}{\omega_{o}}\right| \ll 1 .
$$

The highest audible frequency is around $20 \mathrm{kHz}$, whereas the light oscillates at frequencies of the order of terahertz. Therefore, this condition is clearly fulfilled when analyzing the acousto-optic effect in the audible frequency range.

The exact solution to the electromagnetic wave equation in the presence of sound can be derived by inserting Eq. (7) into the wave equation

$$
\begin{aligned}
& \mathbf{E}\left(j \frac{\partial^{2} \phi}{\partial z^{2}}-\left(\frac{\partial \phi}{\partial z}\right)^{2}\right) \\
& -\left(\frac{n}{c_{0}}\right)^{2} \mathbf{E}\left(j \frac{\partial^{2} \phi}{\partial t^{2}}-\left(\omega_{0}+\frac{\partial \phi}{\partial t}\right)^{2}\right)=0 .
\end{aligned}
$$

Without losing generality, it is assumed that the light travels along the $z$-direction. According to Eq. (B1), the temporal variation of $\phi$ can be regarded to be much slower than the angular frequency of the electromagnetic field

$$
\left|\frac{\partial \phi}{\partial t}\right| \ll \omega_{o} \quad \text { and } \quad\left|\frac{\partial^{2} \phi}{\partial t^{2}}\right| \ll \omega_{o}^{2}
$$

Equation (B2) now reduces to

$$
j \frac{\partial^{2} \phi}{\partial z^{2}}-\left(\frac{\partial \phi}{\partial z}\right)^{2}+\left(\frac{n \omega_{0}}{c_{0}}\right)^{2}=0
$$

If the second partial derivative of $\phi(z, t)$ as a function of $z$ is much smaller in absolute value than the square of the first derivative

$$
\left|\frac{\partial^{2} \phi}{\partial z^{2}}\right| \ll\left(\frac{\partial \phi}{\partial z}\right)^{2},
$$

Eq. (B2) can be further simplified

$$
\left(\frac{\partial \phi}{\partial z}\right)^{2}=\left(\frac{n \omega_{o}}{c_{0}}\right)^{2} \Leftrightarrow \frac{\partial \phi}{\partial z}= \pm \frac{n \omega_{o}}{c_{0}}
$$

and therefore $\phi$ can be found by integrating the refractive index along the propagation path

$$
\phi=\phi_{0}+ \pm \frac{\omega_{0}}{c_{0}} \int_{z_{1}}^{z_{2}} n \mathrm{~d} z=\phi_{0} \pm k_{0} \int_{z_{1}}^{z_{2}} n \mathrm{~d} z
$$

Here $z_{1}$ and $z_{2}$ are the initial and final positions of the integration, $\phi_{0}$ is simply an integration constant that depends on the used reference system, and the sign must be chosen according to the direction of propagation, that is, "-" for waves propagating in the positive direction of $z$ and "+" otherwise.

\footnotetext{
${ }^{1}$ D. Royer and O. Casula, "Quantitative imaging of transient acoustic fields by optical heterodyne interferometry," in Proceedings of the 1994 IEEE Ultrasonics Symposium (1994), Vol. 2, pp. 1153-1162.

${ }^{2}$ T. A. Pitts and J. F. Greenleaf, "Three-dimensional optical measurement of instantaneous pressure," J. Acoust. Soc. Am. 108, 2873-2883 (2000).
} 
${ }^{3}$ J. P. Remenieras, O. B. Matar, S. Calle, and F. Patat, "Acoustic pressure measurement by acousto-optic tomography," in Proceedings of the 2001 IEEE Ultrasonics Symposium (2001), Vol. 1, pp. 505-508.

${ }^{4}$ A. R. Harland, J. N. Petzing, and J. R. Tyrer, "Nonperturbing measurements of spatially distributed underwater acoustic fields using a scanning laser Doppler vibrometer," J. Acoust. Soc. Am. 115, 187-195 (2004).

${ }^{5}$ J. M. Buick, J. A. Cosgrove, P.-A. Douissard, C. A. Greated, and B. Gilabert,, "Application of the acousto-optic effect to pressure measurements in ultrasound fields in water using a laser vibrometer," Rev. Sci. Instrum. 75, 3203-3207 (2004).

${ }^{6}$ P. D. Theobald, S. P. Robinson, G. Hayman, and T. Koukoulas, "Acoustooptic tomography for mapping of high-frequency sonar fields," in Proceedings of Acoustics 08 (2008), pp. 2833-2838.

${ }^{7}$ L. Bahr and R. Lerch, "Beam profile measurements using light refractive tomography," IEEE Trans. Ultrason. Ferroelectr. Freq. Control 55, 405-414 (2008).

${ }^{8}$ X. Jia, G. Quentin, and M. Lassoued,, "Optical heterodyne detection of pulsed ultrasonic pressures," IEEE Trans. Ultrason. Ferroelectr. Freq. Control 40, 67-69 (1993).

${ }^{9}$ K. Nakamura, M. Hirayama, and S. Ueha, "Measurements of air-borne ultrasound by detecting the modulation in optical refractive index of air," in Proceedings of the 2002 IEEE Ultrasonics Symposium (2002), pp. 609-612.

${ }^{10}$ Y. Oikawa, M. Goto, Y. Ikeda, T. Takizawa, and Y. Yamasaki, "Sound field measurements based on reconstruction from laser projections," in
IEEE International Conference on Acoustics, Speech and Signal Processing (2005), pp. 661-664).

${ }^{11}$ T. Sakoda and Y. Sonoda, "Visualization of sound field with uniform phase distribution using laser beam microphone coupled with computerized tomography method," Acoust. Sci. \& Tech. 29, 295-299 (2008).

${ }^{12}$ Y. Oikawa, T. Hasegawa, Y. Ouchi, Y. Yamasaki, and Y. Ikeda, "Visualization of sound field and sound source vibration using laser measurement method," in Proceedings of the 20th International Congress on Acoustics (2010).

${ }^{13}$ A. D. Pierce, Acoustics: An Introduction to its Physical Principles and Applications (Acoustical Society of America, Melville, New York, 1989), Chap. 1.

${ }^{14}$ J. H. Gladstone and T. P. Dale, "Researches on the refraction, dispersion, and sensitiveness of liquids," Philos. Trans. R. Soc. London 153, 317-343 (1863).

${ }^{15}$ W. Merzkirch, Techniques of Flow Visualization (Advisory Group for Aerospace Research \& Development, Loughton, Essex, 1987).

${ }^{16} \mathrm{P}$. M. Morse and K. U. Ingard, Theoretical Acoustics (Princeton University Press, Princeton, New Jersey, 1986), Chap. 13.

${ }^{17} \mathrm{~A}$. Torras-Rosell, S. Barrera-Figueroa, and F. Jacobsen, "An investigation of sound fields based on the acousto-optic effect," in Proceedings of 18th International Congress on Sound \& Vibration (2011).

${ }^{18}$ A. C. Kak and M. Slaney, Principles of Computerized Tomographic Imaging (IEEE, New York, 1988), Chaps. 3 and 5. 